\title{
Perceived Event Frequency and the Optimistic Bias: Evidence for a Two-Process Model of Personal Risk J udgments
}

\author{
Paul C. Price, Heather C. Pentecost, and Rochelle D. Voth, California State University, Fresno
}

\begin{abstract}
People often judge themselves to be at lower risk for various negative life events than are their peers. The two empirical studies presented here show that the magnitude of this optimistic bias can be either negatively or positively related to the perceived frequency of the event, depending on whether people judge their own risk relative to that of an average peer (make comparative risk judgments) or judge their own and an average peer's risk separately (make absolute risk judgments). A new two-process model is presented to account for these results. The model combines a better-thanaverage heuristic with elements of the singular target-focused and singular-distributional models of Klar and colleagues (Klar \& Giladi, 1997, 1999; Klar, Medding, \& Sarel, 1996). The empirical results and model have many implications for the study of personal risk judgments, the optimistic bias, and risk-taking behavior.
\end{abstract}

When people judge their risk of experiencing negative life events such as becoming alcoholic, developing cancer, being injured in a car crash, and getting divorced, they generally exhibit an optimistic bias. That is, the vast majority of people judge their own risk to be lower than that of their average peer (Weinstein, 1980). Theoretical and empirical research on this phenomenon has focused on a number of moderating variables including the perceived desirability of the event (e.g., Weinstein, 1980, 1987), the perceived controllability of the event (Harris, 1996; Klar, Medding, \& Sarel, 1996; McKenna, 1993), and personal experience with the event (e.g., Burger \& Palmer, 1992; Weinstein, 1987). In fact, there is reasonably good evidence that each of these variables plays some role in the optimistic bias. The current research, however, focuses on another moderating variable: the perceived frequency of the event. Although there is little question that the perceived frequency of the event is related to the magnitude of the optimistic bias, there is, surprisingly, some disagreement about the direction of this relationship. Some research suggests that events that are perceived as less frequent elicit a larger optimistic bias, whereas other research suggests that

This research was supported in part by a grant from the Decision, Risk, and Management Science Program, National Science Foundation.

Address correspondence and reprint requests to Paul C. Price, Department of Psychology, California State University, Fresno, 5310 North Campus Drive, Fresno, CA 93740-8019. E-mail: paulpri@csufresno.edu. events that are perceived as more frequent elicit a larger optimistic bias.

There are several reasons that this is an interesting and important problem. One is that it might have implications for the field of health and accident risk communication. It might be helpful for researchers and practitioners in this field to know whether people exhibit a larger optimistic bias for rare or common events, and under what conditions, so that they can develop ways to communicate risk information more effectively. Another reason is that, as detailed presently, the direction of the relationship between event frequency and the optimistic bias seems to depend on the method used to elicit people's risk judgments. The current research, therefore, might elucidate important connections between method and theory in the study of personal risk judgments. Perhaps the most important reason, however, is that making sense of this apparent contradiction might help researchers to develop more complete theories and models of personal risk judgments and the optimistic bias. Consider, for example, that in the domain of health, the perceived prevalence of a disorder has a negative effect on the perceived seriousness of that disorder (Jemmott, Croyle, \& Ditto, 1988; Jemmott, Ditto, \& Croyle, 1986). For this reason, understanding the relationship between event frequency and the optimistic bias might lead to a better understanding of the relationship between event seriousness and the optimistic bias - a relationship that is particularly im- 
portant in motivational theories of the phenomenon (Weinstein, 1987).

\section{A Negative Frequency-Optimism Relationship for Comparative Risk Judgments}

Weinstein (1980) was the first to suggest, and to confirm empirically, that the perceived frequency of an event is negatively correlated with the magnitude of the optimistic bias. In his procedure, subjects made comparative risk judgments. That is, they rated their own risk relative to that of their peers on a 7-point scale. Judgments at the midpoint of the scale indicated that one's own risk was equal to that of one's average peer, judgments below the midpoint of the scale indicated that one's own risk was less than that of one's average peer, and judgments above the midpoint of the scale indicated that one's own risk was greater than that of one's average peer. Weinstein found that subjects' mean comparative risk judgment fell farther below the midpoint of the scale for events that, on average, were perceived to be relatively infrequent. That is, the magnitude of the optimistic bias was larger for less frequent events. Later, in combining three separate data sets, Weinstein (1987) found that the correlation between the perceived frequency of an event and the magnitude of the optimistic bias was -.33 . $^{1}$

From a normative perspective, this result makes little sense. The fact that a negative event is rare might imply that one's own risk is low and that one's peers' risk is low, but it implies nothing about one's own risk relative to one's peers' risk. From a psychological perspective, however, this result is consistent with the idea that people give minimal consideration to their peers' risk (Klein \& Weinstein, 1997; Weinstein, 1980). Instead, they might apply a better-thanaverage heuristic (e.g., Alicke, Klotz, Breitenbecher, Yurak, \& Vredenburg, 1995) and assume that they are at lower than average risk. Good evidence for this proposition in the domain of personal risk judgments comes from the work of Rothman, Klein, and Weinstein (1996), who found that people tend to judge their probability of experiencing a negative health event to be just below the stated prevalence of the event, even as the stated prevalence was manipulated to be higher or lower than the actual value.

But how much lower than average do people judge themselves to be? We suggest that this depends on how low they perceive their own absolute risk to be. If they perceive their own absolute risk to be somewhat low, then they judge it to be somewhat below average. If they perceive their own absolute risk to be extremely low, then they judge it to be far below average. Klar and Giladi $(1997,1999)$ have recently formalized some of these ideas in their singular targetfocused (STF) model of comparative risk judgments, and

\footnotetext{
${ }^{1}$ As Weinstein (1987) pointed out, however, the perceived frequency of an event tended to be intercorrelated with direct experience with the event, which complicates the interpretation of these results.
}

they have used this model to explain why people judge themselves - or any individual - to be better than average on a variety of dimensions (for a similar analysis, see also Kruger, 1999). For current purposes, it is important to note that this implies that if people's perceptions of their own risk are at all positively related to the frequency of the event, then less frequent events should produce greater optimistic biases.

\section{A Positive Frequency-Optimism Relationship for Absolute Risk Judgments}

Note, however, that the optimistic bias in personal risk judgments is also observed when people make absolute judgments of their own risk and their average peer's risk (e.g., Klar et al., 1996; Otten \& van der Pligt, 1996; Rothman et al., 1996). The STF model does not apply to this situation because people are forced to consider both themselves and their peers explicitly. Interestingly, this is also the situation in which existing theory and data suggest that the relationship between event frequency and the optimistic bias reverses; it becomes positive rather than negative. Consider first the suggestion of Klar et al. (1996) that people think about their own risk and their average peer's risk in very different ways. Specifically, they think about their own risk in a singular mode, and they think about their average peer's risk in a distributional mode. That is, they think about their own risk in terms of the characteristics they exhibit and the behaviors they engage in that affect their risk for the event, but they think about their average peer's risk in terms of the frequency or relative frequency of the event in some relevant population. Note that this is consistent with the idea that people often neglect to consider base rate information when making judgments about individuals (Kahneman \& Tversky, 1973). For example, a woman might think about her own risk of eventually getting divorced in terms of her beliefs about the sanctity of marriage, how wonderful her spouse is, and so forth. But she might think about her average peer's risk in terms of the relative frequency of divorce in the general population (e.g., "I've heard that half of all marriages end in divorce"). Note that this singulardistributional theory implies that people's beliefs about their own risk should not depend on the perceived frequency of the event but that their beliefs about their average peer's risk should depend heavily on it. As the perceived frequency of the event increases, therefore, people's beliefs about their own risk should remain constant, but their beliefs about their average peer's risk should increase. If the optimistic bias is defined as the discrepancy between the two judgments, then the optimistic bias should increase as the perceived frequency of the event increases.

We have conducted a first test of this prediction by reanalyzing some unpublished data collected in our laboratory for other purposes (Voth \& Price, 1999). In that study, 


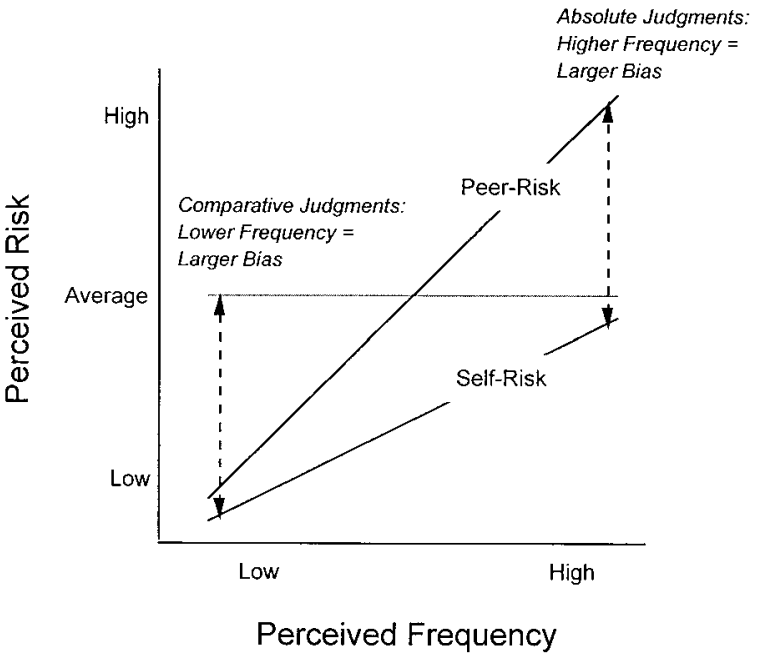

FIG. 1. Two-process model of comparative and absolute risk judgments.

205 college students estimated the percentage of Americans who would experience each of 10 negative life events, including becoming alcoholic. Approximately half then judged the probability that they would become alcoholic on a scale from $0 \%$ to $100 \%$, and the rest judged the probability that a randomly selected, but otherwise unspecified, peer would become alcoholic. Note that judgments about a randomly selected peer, like judgments about one's average peer, also produce an optimistic bias (Alicke et al., 1995). Furthermore, it is reasonable to expect people to engage in distributional thinking about such a peer both because it is nonspecific and because the phrase "randomly selected" is likely to encourage it (Ginossar \& Trope, 1987; Kahneman \& Tversky, 1973). Of interest here is that participants' frequency judgments were more strongly correlated with their peer-risk judgments $(r=.54)$ than with their self-risk judgments $(r=.39)$. Furthermore, the slope of the regression line relating peer-risk judgments to perceived frequency $(b=0.45)$ was greater than that relating self-risk judgments to perceived frequency $(b=0.33), t(201)=$ $2.38, p<.05$.

\section{A Two-Process Model}

There are plausible explanations, then, both for the negative relationship between perceived frequency and the optimistic bias measured using comparative risk judgments and for the proposed positive relationship between perceived frequency and the optimistic bias measured using absolute risk judgments. An important question is whether it is possible to integrate these two explanations into a single, internally consistent model. We think that it is, and Fig. 1 summarizes our attempt at such an integration.

Our two-process model assumes that both self-risk judgments and peer-risk judgments are positively related to perceived event frequency but that peer-risk judgments are more strongly related to it (in the sense of having a greater slope). When people make separate self-risk judgments and peer-risk judgments, therefore, the difference between those judgments increases as a function of the perceived event frequency. In Fig. 1, this is shown by the increasing separation of the two solid lines as a function of perceived frequency. When people make comparative risk judgments, however, somewhat different processes are involved. First, they do not have to judge the risk of an average peer because it is given to them by the experimenter, who might label the midpoint of a 7-point risk scale as average risk. People's task, then, is simply one of judging their own risk relative to the average risk. To do so, they use a better-thanaverage heuristic, anchoring their judgments on the risk of an average peer but adjusting downward. Furthermore, they adjust downward to the extent that they judge themselves to be at low absolute risk. When people make comparative risk judgments, therefore, the extent to which they judge themselves below average increases with decreasing perceived frequency. In Fig. 1, this is shown by line representing people's self-risk judgments falling farther below average (the horizontal dashed line) as the perceived frequency of the event decreases.

One additional theoretical clarification may be in order here. The current two-process model (and the empirical results of Voth \& Price, 1999) seems inconsistent with an important assumption of the singular-distributional model of Klar et al. (1996). That model assumes that people do not use frequency information at all to make self-risk judgments (or risk judgments about any individual). This, in turn, seems to imply that perceived event frequency should not be related to people's self-risk judgments. It is possible, of course, that the assumption of Klar et al. is incorrect. People might use frequency information to make self-risk judgments, but perhaps they do not weight it as heavily as when they make peer-risk judgments. Another possibility is that people do not use frequency information to make self-risk judgments but that their self-risk judgments actually influence their frequency judgments. People who perceive themselves at high risk for a negative event based on their personal characteristics and behaviors alone may infer that the event is relatively frequent (cf. Klein, 1997). This would also result in a positive relationship between perceived event frequency and self-risk judgments. Yet another possibility is that both perceived event frequency and self-risk judgments are influenced by a third variable: the availability in memory of behaviors and characteristics that increase one's risk. The key point is that this relationship is a crucial element of the two-process model, however one chooses to explain it.

\section{The Current Studies}

The current studies were conducted to test more directly the hypothesis that the relationship between event frequency 
and the optimistic bias for negative life events depends on the method used to elicit people's risk judgments and, therefore, to test the two-process model proposed here. Again, we hypothesized that when people make comparative risk judgments (i.e., of their own risk relative to that of their average peer), the relationship between event frequency and the optimistic bias is negative. This is because comparative risk judgments fall below average to the extent that people perceive themselves to be at low absolute risk. We also hypothesized that when people make absolute risk judgments (i.e., of their own risk and their average peer's risk), the relationship between event frequency and the optimistic bias is positive. This is because people's judgments of their peers' risk are based on event frequency to a greater extent than are their judgments of their own risk, so the discrepancy between self-risk and peer-risk judgments increases as event frequency increases.

\section{STUDY 1}

In Study 1, participants estimated the relative frequency of 20 negative life events and then made personal risk judgments for each of those 20 events. In the comparative judgment condition, they judged their own risk relative to that of an average peer on a 7-point risk scale. In the absolute judgments condition, they judged their own risk and an average peer's risk, also on 7-point scales. The goal was to see whether the direction of the relationship between event frequency and the optimistic bias would be negative in the former condition and positive in the latter, while controlling as many confounding variables as possible. This research design also allowed us to test the proposed twoprocess model in two additional ways. First, the model predicts that, across the 20 events, the mean comparative risk judgment should be highly correlated with the mean self-risk judgment. This is because it holds that comparative risk judgments are low to the extent that self-risk judgments are low (cf. Klar \& Giladi, 1997; 1999; Kruger, 1999). Second, the model predicts that event frequency should be more strongly related to people's peer-risk judgments than to their self-risk judgments. This might be because people rely more heavily on base rate information when thinking about groups or statistical summaries of groups than when thinking about individuals (Klar et al., 1996).

\section{Method}

Participants. The participants were 78 students at California State University, Fresno. They participated in fulfillment of an introductory psychology course requirement.

Materials. Participants completed two questionnaires. The first required them to estimate the percentage of Americans who experience each of 20 different negative life events (see Table 1). For each event, they responded by circling one of 11 different percentages $(1 \%, 10 \%, 20 \%$, $\ldots, 100 \%)$, which were arrayed horizontally beneath the description of the event. The first item, for example, read as follows:

1. Percentage of Americans that eventually Commits Suicide:

$1 \%$ 10\% 20\% 30\% 40\% 50\% 60\% 70\% $80 \%$ 90\% $100 \%$

Next, participants completed a risk judgment questionnaire. In the comparative judgment condition $(n=40)$, participants made a direct comparative risk judgment for each event. They responded by indicating which of seven statements best described their chances of experiencing the event relative to other students their age and sex. The seven statements were arrayed vertically below the comparative risk question. The first item, for example, read as follows:

Compared to other Fresno State students my age and sex, my chances of Committing Suicide are:

- much below average

- below average

- a little below average

- average

- a little above average

- above average

- much above average

For half of these participants, the seven response statements were arrayed exactly as above, with much below average at the top and much above average at the bottom. For the rest, the order of the response statements was reversed. This was intended to ensure that any observed relationship between perceived frequency and the optimistic bias did not reflect a simple position preference in the use of the response scale.

Participants in the absolute judgments condition $(n=38)$ were asked first to judge the chances that the average student at their institution would experience each event. They responded by indicating which of seven statements best described their average peer's chances of experiencing the event. The seven statements, which were arrayed vertically below the absolute risk question, were extremely low, very low, somewhat low, moderate, somewhat high, very high, and extremely high. Again, the order of the seven statements was reversed for half of the participants. These participants were then asked to judge the chances that they would experience each event. They responded using the same response scale-with the response statements in the same order-as for their judgments about their average peer. The order of the seven response statements was the same as it was for their judgments about their average peer.

\section{Results and Discussion}

In the comparative judgment condition, we coded the responses 1 (much above average) through 7 (much below 
TABLE 1

Means and Standard Deviations of Relative Frequency Judgments and Optimistic Biases, and the Correlations between Those Variables for 20 Events under Each of Two Judgment Elicitation Conditions for Experiment 1

\begin{tabular}{|c|c|c|c|c|c|c|}
\hline \multirow[b]{3}{*}{ Event } & \multicolumn{6}{|c|}{ Condition } \\
\hline & \multicolumn{3}{|c|}{ Comparative judgment } & \multicolumn{3}{|c|}{ Absolute judgments } \\
\hline & Frequency & Optimism & $r$ & Frequency & Optimism & $r$ \\
\hline Limb amputated & $13.82(14.20)$ & $4.50(1.45)^{*}$ & -.16 & $13.47(12.43)$ & $0.59(0.98)^{*}$ & .12 \\
\hline Suicide & $18.15(12.23)$ & $5.30(1.29)^{*}$ & -.19 & $18.37(14.03)$ & $1.00(1.14)^{*}$ & .28 \\
\hline House fire & $22.76(20.61)$ & $3.62(1.13)^{*}$ & -.07 & $22.21(14.20)$ & $0.00(1.18)$ & .19 \\
\hline AIDS & $26.83(20.46)$ & $5.00(1.04)^{*}$ & -.20 & $23.30(15.38)$ & $1.82(1.52)^{*}$ & $.34 *$ \\
\hline Gum disease & $30.58(22.58)$ & $4.25(1.43)^{*}$ & $-.35^{*}$ & $28.18(18.68)$ & $0.74(1.31)^{*}$ & -.03 \\
\hline Hearing loss & $33.10(24.27)$ & $3.90(1.63)^{*}$ & .01 & $29.76(18.63)$ & $0.63(1.32)^{*}$ & .21 \\
\hline Death by car crash & $30.38(20.00)$ & $3.10(0.78)$ & $-.46^{*}$ & $32.89(17.54)$ & $0.42(1.24)^{*}$ & -.02 \\
\hline Sued & $37.53(21.53)$ & $4.12(1.22)^{*}$ & $-.38^{*}$ & $34.76(20.05)$ & $0.87(1.32)^{*}$ & -.13 \\
\hline Cancer & $37.00(21.51)$ & $3.37(1.17)^{*}$ & -.30 & $37.11(19.58)$ & $0.18(0.95)$ & -.04 \\
\hline Alcoholism & $40.25(17.61)$ & $4.67(1.70)^{*}$ & -.02 & $37.63(18.37)$ & $2.76(1.62)^{*}$ & .12 \\
\hline Heart attack & $36.25(19.83)$ & $4.02(1.54)^{*}$ & $-.50 *$ & $45.26(20.10)$ & $0.29(1.63)$ & .05 \\
\hline Car stolen & $44.05(25.71)$ & $2.97(1.46)$ & $-.51^{*}$ & $38.95(22.75)$ & $0.82(1.25)^{*}$ & -.01 \\
\hline Death before 65 years of age & $41.34(21.66)$ & $3.40(1.22)^{*}$ & -.01 & $42.37(18.52)$ & $0.70(1.60)^{*}$ & .01 \\
\hline Drops out of college & $41.34(23.22)$ & $4.60(1.61)^{*}$ & -.14 & $45.79(16.71)$ & $2.65(1.60)^{*}$ & $.44^{*}$ \\
\hline Depression & $43.83(27.95)$ & $3.55(1.72)^{*}$ & $-.36^{*}$ & $44.74(24.02)$ & $1.34(1.65)^{*}$ & .18 \\
\hline Burglarlized & $42.52(26.15)$ & $3.40(1.53)$ & $-.43^{*}$ & $48.68(25.49)$ & $0.50(1.41)^{*}$ & .15 \\
\hline Fired from job & $44.50(21.95)$ & $3.92(1.21)^{*}$ & -.10 & $49.47(22.17)$ & $1.58(1.43)^{*}$ & .03 \\
\hline Bad career choice & $52.89(25.03)$ & $3.25(1.66)$ & -.31 & $56.05(18.97)$ & $1.62(1.30)^{*}$ & .25 \\
\hline Broken bone & $49.79(23.88)$ & $3.17(1.36)$ & -.06 & $59.21(23.52)$ & $0.76(1.63)^{*}$ & $.40^{*}$ \\
\hline Divorce & $55.00(17.97)$ & $3.87(1.65)^{*}$ & -.23 & $55.53(16.39)$ & $1.61(1.69)^{*}$ & $-.43 *$ \\
\hline
\end{tabular}

Note. Events are in ascending order of mean judged frequency across all 78 participants.

$* p<.05$.

average). We did this so that larger numbers would correspond to a larger optimistic bias. In the absolute judgments condition, we coded the responses 1 (extremely high) through 7 (extremely low). Then we subtracted each participant's judgment about himself or herself from that participant's judgment about his or her average peer. We did this, again, so that larger numbers would correspond to a larger optimistic bias. The means and standard deviations of the optimistic bias for each event, and for each judgment elicitation condition, are presented in Table 1. Note that the majority of events produced optimistic biases under both judgment elicitation conditions. In the comparative judgment condition, the mean judgment tended to be less than 4 (corresponding to below average risk). In the absolute judgments condition, the mean peer-self difference tended to be greater than zero (corresponding to a lower judgment for oneself than for one's peers).

Before proceeding, it is worth noting that it is impossible to say whether individual participants are optimistically biased without an objective measure of each participant's risk (Klein \& Weinstein, 1997). For this reason, the primary correlational analyses reported here used events as the unit of analysis. Additional analyses with participants as the unit of analysis are also reported, however, to confirm the basic result.

We computed the mean perceived frequency for each event across the 40 participants in the comparative judgment condition and across the 38 participants in the absolute judgments condition (see Table 1). Then we examined the relationship between the mean perceived frequency and the mean optimistic bias across the 20 events, separately by condition (see Fig. 2). As hypothesized, the correlation between these two variables was negative in the comparative judgment condition $(r=-.53, p<.05)$ and positive in

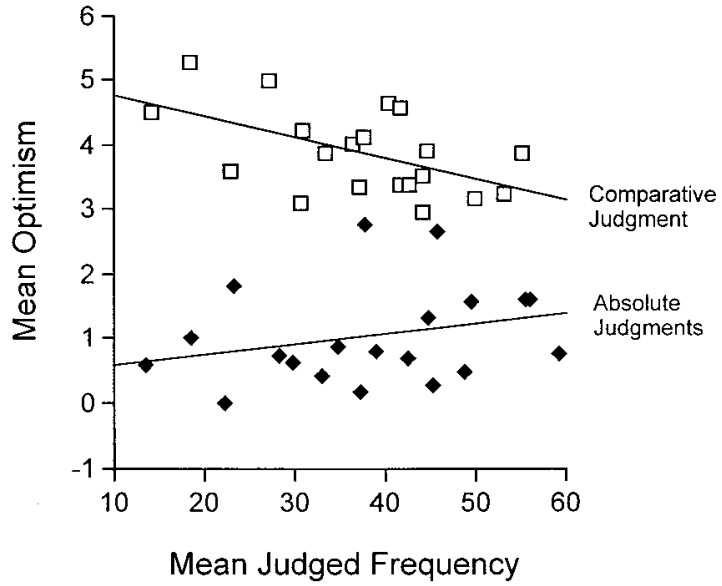

FIG. 2. Relationship between mean perceived frequency and mean optimistic bias for 20 negative life events, for both comparative risk judgments and absolute risk judgments, in Study 1. 


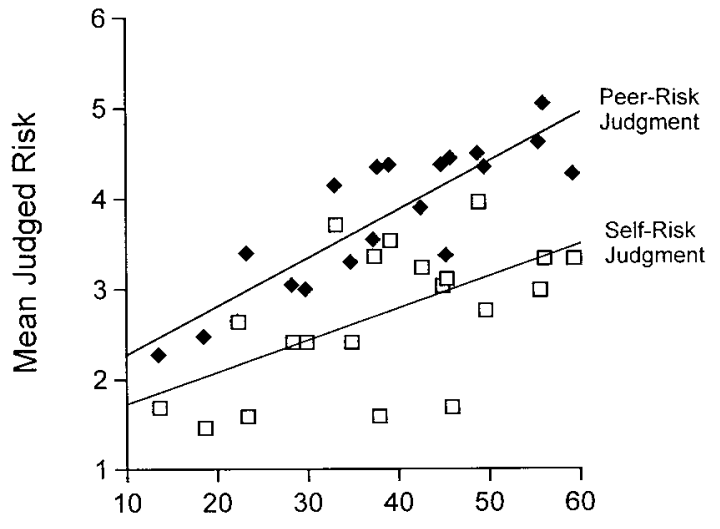

Mean Judged Frequency

FIG. 3. Relationship between mean perceived frequency and both mean self-risk judgments and mean peer-risk judgments for 20 negative life events in Study 1.

the absolute judgments condition $(r=.31, p=.18)$. The difference between these two correlations was statistically significant, $t(17)=3.72, p<.005 .^{2}$

Recall that the two-process model also predicts that the mean comparative risk judgment should be highly correlated with the mean self-risk judgment but not highly correlated with the mean peer-risk judgment. In fact, these correlations were $.93(p<.001)$ and $.49(p<.05)$, respectively. ${ }^{3}$ Not surprisingly, a multiple regression analysis with the mean self-risk and peer-risk judgments as independent variables showed that only the mean self-risk judgment was a statistically significant predictor of the mean comparative judgment $(b=0.78, S E=0.09, t=8.90, p<.001)$. The mean peer-risk judgment was not $(b=0.02, S E=0.09, t=$ $0.18, p=.86)$. Recall also that the two-process model predicts that the mean perceived frequency should be more strongly related to the mean peer-judgment than to the mean self-judgment. That this was the case is shown clearly in Fig. 3. The correlation between the mean perceived frequency and the mean peer-risk judgment was .87 ( $p<$ $.001)$. That between the mean perceived frequency and the mean self-risk judgment was $.59(p<.01)$. The difference between these two correlations was statistically significant, $t(35)=2.52, p<.05$.

We also analyzed the relationship between perceived frequency and the perceived self-peer risk difference separately for each event using participants as the unit of analysis. Table 1 presents the correlation coefficients for each event and for each of the two judgment elicitation condi-

\footnotetext{
${ }^{2}$ To test the difference between these correlations, we used the test for dependent samples described by Cohen and Cohen (1983). The samples are dependent because the unit of analysis is the event, and the two correlations were computed across the same 20 events.

${ }^{3}$ Keep in mind that these correlations can be quite high because they are correlations between means computed across participants.
}

tions. In the comparative judgment condition, the relationship was negative for 18 of the 20 events $(p<.05$ by an exact binomial test). In the absolute judgments condition, the correlation was positive for 14 of the 20 events $(p=.12$ by an exact binomial test). This strongly suggests that this is not just an aggregate phenomenon or one that depends on the specific events chosen for study.

Taken together, these results support the hypothesis that the direction of the relationship between event frequency and the optimistic bias depends on the method used to elicit people's risk judgments. They also support the two-process model proposed here.

\section{STUDY 2}

The primary purpose of Study 2 was to replicate the results of Study 1, maintaining the basic difference between the comparative judgment and absolute judgments conditions but changing several other aspects of the method used to elicit people's risk judgments. The most important change was that all risk judgments were expressed as percentages. In the comparative judgment condition, participants judged their own risk for each event as a percentage of the average student's risk (cf. Weinstein, 1980). In the absolute judgments condition, participants judged their own probability and the average student's probability on a scale of $0 \%$ to $100 \%$ (cf. Rothman et al., 1996). This was done to be sure that the effect observed in Study 1 did not depend on the 7-point risk judgment scale. Consider that judgments on a 7-point scale can be construed by participants as concerning aspects of risk other than the likelihood of the event (Weinstein \& Nicolich, 1993). Also, when participants perceive very small differences in risk, such a crude rating scale necessarily obscures or inflates them (Gerrard, Gibbons, \& Bushman, 1996). We also counterbalanced the order in which participants make their frequency and risk judgments and the order in which participants in the absolute judgments condition made their self- and peer-risk judgments. Again, we did this to be sure that the results of Study 1 did not depend on these kinds of procedural details.

A secondary purpose of Study 2 was to ask participants to judge the seriousness of each event and to see how event seriousness relates to both event frequency and the optimistic bias. Recall that in the domain of health, less common disorders are generally perceived to be more serious (Jemmott et al., 1986, 1988). This suggests that the relationship between event seriousness and the optimistic bias might also depend on the judgment elicitation method.

\section{Method}

Participants. The participants were 79 students at California State University, Fresno. They participated in fulfillment of an introductory psychology course requirement. 
Materials. Again, participants made frequency judgments and risk judgments for 20 negative life events. The events were the same as in Study 1, but they were presented in a different randomized order. Unlike in Study 1, the order of the judgments was counterbalanced, such that half of the participants made their frequency judgments before their risk judgments, while the rest made their risk judgments before their frequency judgments. The frequency judgment questionnaire required participants to judge the frequency with which Americans experience the 20 events. They rated the frequency of each event on a 9-point scale. The integers 1 to 9 were arrayed horizontally from left to right, with the left end of the scale labeled as extremely infrequent and the right end labeled as extremely frequent. Participants responded by circling one of the integers. Note that we changed the frequency judgment scale from the percentage scale used in Study 1 so that participants could not directly translate their frequency judgments into risk judgments or vice versa.

In completing the risk questionnaire, participants in the comparative judgment condition $(n=40)$ judged their own risk for each event as a percentage of the risk of the average student at their university (Weinstein, 1980). They responded by writing a percentage, anywhere from $0 \%$ upward, for each event. For example, a judgment of $100 \%$ meant that they thought their risk was the same as that of the average student, a judgment of 50\% meant that they thought their risk was half that of the average student, and a judgment of $200 \%$ meant that they thought their risk was twice that of the average student. To help participants make this judgment, a visual analog of the response scale appeared at the top of each page of the questionnaire. This was a horizontal line with percentages from $0 \%$ to $200 \%$ labeled at intervals of $10 \%$ and percentages from $200 \%$ to $500 \%$ labeled at intervals of $50 \%$. An additional label, average student, appeared above the $100 \%$ mark on the response scale.

Participants in the absolute judgments condition $(n=39)$ judged the probability that they would experience each event, and the probability that the average student at their university would experience each event, on a scale from $0 \%$ to $100 \%$. They responded by writing a percentage from $0 \%$ to $100 \%$ for each event. Again, to help participants make this judgment, we presented a visual analog of the response scale on each page of the questionnaire. This was a horizontal line with probabilities from $0 \%$ to $1 \%$ labeled at intervals of $0.1 \%$, probabilities from $1 \%$ to $10 \%$ labeled at intervals of $1 \%$, and probabilities from $10 \%$ to $100 \%$ labeled at intervals of $10 \%$. This was done in part to encourage participants to use small probabilities-including probabilities less than 1\%-when they thought it appropriate (Rothman et al., 1996). Half of the participants in the absolute judgments condition made self-risk judgments followed by peer-risk judgments, while the rest made peer-risk judgments followed by self-risk judgments. This counterbalancing was done because judgment order has been shown to influence the magnitude of the optimistic bias (Otten \& van der Pligt, 1996).

The last thing that all participants did was to complete a seriousness judgment questionnaire. This questionnaire required them to rate the seriousness of each of the 20 events on a 7-point scale. The integers 1 to 7 were arrayed horizontally, from left to right, beneath the description of each event. The left end of the scale was labeled not serious, and the right end of the scale was labeled extremely serious. Participants responded by circling one of the integers for each event.

\section{Results and Discussion}

In the comparative judgment condition, we subtracted each judgment from $100 \%$ so that larger values would correspond to a larger optimistic bias. In the absolute judgments condition, we subtracted each participant's judgments about himself or herself from that participant's judgments about his or her average peer. We did this, again, so that larger numbers would correspond to a larger optimistic bias. The means and standard deviations of the optimistic bias for each event, and for each judgment elicitation condition, are presented in Table 2. Note that, again, the majority of events produced optimistic biases under both judgment elicitation conditions. In the comparative judgment condition, the mean optimistic bias tended to be positive (corresponding to below average risk for oneself), and in the absolute judgments condition, the mean peer-self difference tended to be positive (corresponding to a lower judgment for oneself than for one's average peer).

We computed the mean perceived frequency for each event across the 40 participants in the comparative judgment condition and across the 39 participants in the absolute judgments condition (see Table 2), and we examined the correlation between the mean perceived frequency and the mean optimistic bias across the 20 events, separately by condition. As hypothesized, the correlation between these variables was negative in the comparative judgment condition $(r=-.62, p<.01)$ and positive in the absolute judgments condition $(r=.50, p<.05)$ (see Fig. 4 ).

Again, two additional results support the two-process model proposed here. First, the mean comparative risk judgment was strongly correlated with the mean self-risk judgment across events $(r=.88, p<.001)$ but less strongly correlated with the mean peer-risk judgment across events $(r=.56, p<.05)$. Furthermore, in a multiple regression analysis, only the mean self-risk judgment was a statistically significant predictor of the mean comparative judgment $(b=2.00, S E=0.35, t=5.78, p<.001)$. The mean peer-risk judgment was not $(b=0.06, S E=0.30, t=0.19$, $p=.85)$. This supports the two-process model, which assumes that comparative risk judgments are low to the 
TABLE 2

Means and Standard Deviations of Frequency Judgments and Optimistic Biases, and the Correlations between Those Variables for 20 Events under Each of Two Judgment Elicitation Conditions for Experiment 2

\begin{tabular}{|c|c|c|c|c|c|c|}
\hline \multirow[b]{3}{*}{ Event } & \multicolumn{6}{|c|}{ Condition } \\
\hline & \multicolumn{3}{|c|}{ Comparative judgment } & \multicolumn{3}{|c|}{ Absolute judgments } \\
\hline & Frequency & Optimism & $r$ & Frequency & Optimism & $r$ \\
\hline Limb amputated & $3.02(1.59)$ & $40.12(62.47)^{*}$ & -.04 & $3.02(1.44)$ & 3.49 (10.97) & .07 \\
\hline Suicide & $3.95(1.89)$ & $78.92(33.45)^{*}$ & $-.32 *$ & $4.08(1.98)$ & $16.13(21.38)^{*}$ & $.34^{*}$ \\
\hline House fire & $4.08(1.55)$ & $23.22(53.34)^{*}$ & -.14 & $3.52(1.43)$ & $1.02(16.00)$ & -.19 \\
\hline AIDS & $4.48(2.05)$ & $65.52(61.02)^{*}$ & $-.32 *$ & $4.42(1.91)$ & $19.72(19.43)^{*}$ & .19 \\
\hline Gum disease & $5.05(1.91)$ & $34.22(51.48)^{*}$ & $-.36^{*}$ & $4.05(2.04)$ & $12.43(15.63)^{*}$ & -.01 \\
\hline Hearing loss & $4.82(1.76)$ & $22.58(81.91)$ & -.06 & $4.28(1.58)$ & $1.97(20.49)$ & .02 \\
\hline Sued & $5.55(2.16)$ & $36.85(51.48)^{*}$ & -.27 & $5.08(2.22)$ & $8.89(17.55)^{*}$ & -.06 \\
\hline Death by car crash & $5.37(1.69)$ & $21.62(51.55)^{*}$ & .03 & $5.18(1.68)$ & 3.85 (19.09) & .03 \\
\hline Cancer & $6.02(1.75)$ & $5.00(79.57)$ & $-.40 *$ & $5.60(1.85)$ & $9.34(18.89)^{*}$ & -.08 \\
\hline Alcoholism & $5.90(1.75)$ & $24.75(112.47)$ & -.27 & $5.42(2.07)$ & $30.86(26.73) *$ & $.45^{*}$ \\
\hline Heart attack & $5.87(1.57)$ & $14.18(70.64)$ & -.11 & $5.68(1.86)$ & $7.85(22.98)^{*}$ & .06 \\
\hline Car stolen & $5.95(2.26)$ & $-7.25(97.29)$ & -.25 & $5.85(2.20)$ & $7.37(23.94)$ & $.52 *$ \\
\hline Death before 65 years of age & $6.02(1.46)$ & $-4.88(78.87)$ & -.22 & $5.30(1.54)$ & $8.58(22.56)^{*}$ & .05 \\
\hline Drops out of college & $5.90(1.92)$ & $50.22(69.35)^{*}$ & $-.31 *$ & $5.40(1.97)$ & $22.09(24.69)^{*}$ & $.55^{*}$ \\
\hline Major depression & $6.05(1.55)$ & $4.08(81.03)$ & $-.34 *$ & $5.40(1.84)$ & $11.68(22.63)^{*}$ & .06 \\
\hline Burglarized & $5.90(1.85)$ & $7.88(84.79)$ & -.05 & $5.25(1.98)$ & $12.47(23.83)^{*}$ & -.12 \\
\hline Fired from job & $6.20(1.51)$ & $27.38(58.77)^{*}$ & -.10 & $5.00(1.96)$ & $16.18(25.43)^{*}$ & .17 \\
\hline Bad career choice & $6.42(1.57)$ & $3.35(69.57)$ & -.13 & $5.95(1.99)$ & $20.14(30.21)^{*}$ & $.32^{*}$ \\
\hline Broken bone & $6.72(2.09)$ & $-1.15(75.46)$ & -.22 & $5.92(2.02)$ & $8.09(29.53)$ & .11 \\
\hline Divorce & $6.75(1.55)$ & $24.50(100.09)$ & -.28 & $6.70(2.09)$ & $37.83(25.20)^{*}$ & .21 \\
\hline
\end{tabular}

Note. Events are in the same order as in Table 1.

$* p<.05$.

extent that people perceive their own risk as low. Second, as Fig. 5 shows, the mean event frequency was more strongly related to the mean peer-risk judgment $(r=.92, p<.001)$ than to the mean self-risk judgment $(r=.59, p<.01)$, $t(36)=3.75, p<.01$. This also supports the two-process model, which assumes that event frequency has a greater effect on peer-risk judgments than on self-risk judgments.

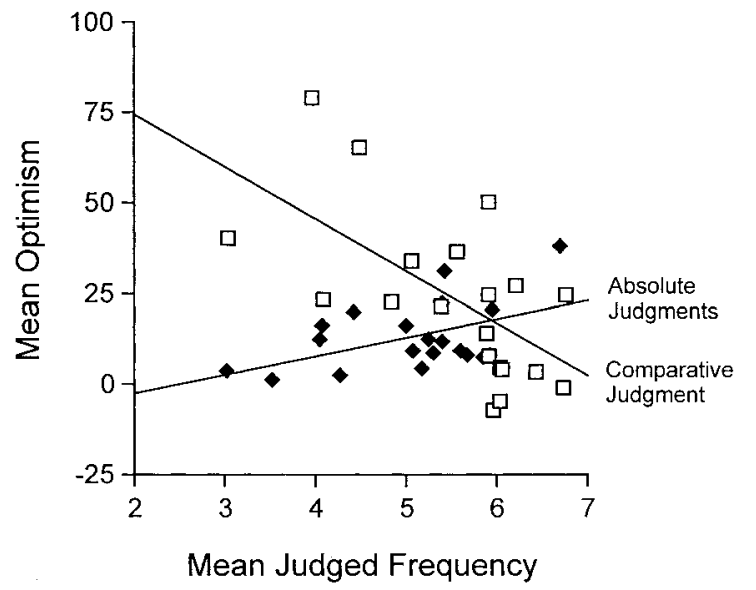

FIG. 4. Relationship between mean perceived frequency and mean optimistic bias for 20 negative life events, for both comparative risk judgments and absolute risk judgments, in Study 2.
This, in turn, explains the positive relationship between event frequency and the optimistic bias for absolute judgments.

Again, we analyzed the relationship between perceived event frequency and the perceived self-peer discrepancy separately for each event using the participant as the unit of analysis. Table 2 presents the correlation coefficients for

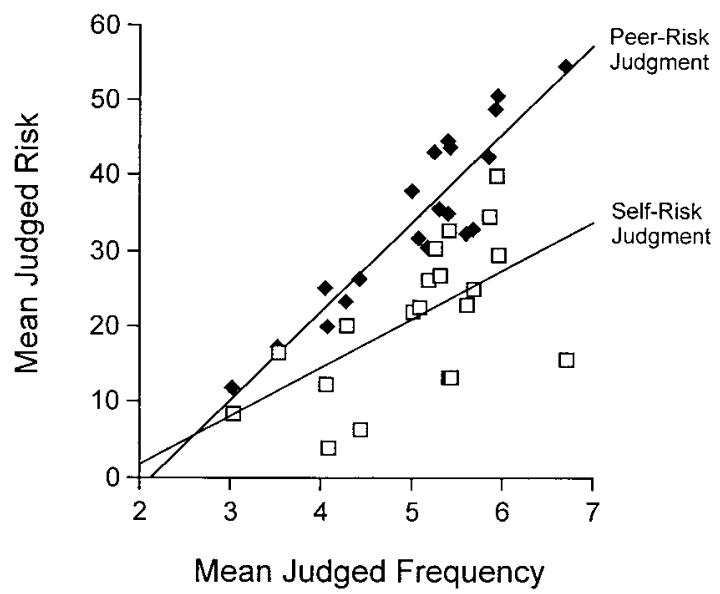

FIG. 5. Relationship between mean perceived frequency and both mean self-risk judgments and mean peer-risk judgments for 20 negative life events in Study 2. 
TABLE 3

Multiple-Regression Analyses of Mean Risk Judgment (comparative, self, and peer) as a Function of Mean Perceived Frequency and Mean Perceived Seriousness across 20 Events

\begin{tabular}{lrrrr}
\hline Risk judgment & $b$ & $S E$ & $B$ & $t$ \\
\hline Comparative & & & & \\
$\quad$ Frequency & 12.16 & 4.38 & .522 & $2.77^{*}$ \\
$\quad$ Seriousness & -8.25 & 5.16 & -.300 & 1.60 \\
Absolute, self & & & & \\
$\quad$ Frequency & 5.47 & 1.87 & .504 & $2.93^{* *}$ \\
$\quad$ Seriousness & -4.62 & 1.90 & -.418 & $2.42^{*}$ \\
Absolute, peer & & & & \\
$\quad$ Frequency & 11.14 & 1.09 & .874 & $10.26^{*}$ \\
$\quad$ Seriousness & -2.70 & 1.11 & -.208 & $2.44^{*}$ \\
\hline
\end{tabular}

$* p<.05$.

$* * p<.01$.

each event and for each of the two judgment elicitation conditions. In the comparative judgment condition, the relationship was negative for 19 of the 20 events ( $p=.000$ by an exact binomial test). In the absolute judgments condition, the correlation was positive for 15 of the 20 events ( $p=$ .041 by an exact binomial test). The basic result, then, was replicated across participants within events.

Event seriousness. As expected, there was a negative correlation between the mean perceived frequency and the mean perceived seriousness across the 20 events $(r=-29$, $p=.22$ ). Events that were perceived as more frequent tended to be perceived as less serious (see also Jemmott et al., 1986, 1988), although this relationship was not statistically significant. In the comparative judgment condition, there was a positive correlation between the mean perceived seriousness and the mean optimistic bias $(r=.47, p=.04)$. A multiple regression analysis with the mean optimistic bias as the dependent variable, and the mean perceived frequency and mean perceived seriousness as independent variables, revealed a statistically significant effect of perceived frequency but a nonsignificant effect of perceived seriousness (see Table 3). It is still not clear, therefore, whether perceived seriousness accounts for some variance in comparative risk judgments independently of perceived frequency.

In the absolute judgments condition, the mean perceived seriousness was negatively correlated with both the mean self-risk judgment $(r=-.52, p<.05)$ and the mean peer-risk judgment $(r=-.39, p=.09)$. Furthermore, multiple regression analyses showed that the mean perceived seriousness made statistically significant contributions to the mean self-risk judgment and the mean peer-risk judgment beyond those of the mean perceived frequency (see Table 3). Interestingly, because the mean perceived seriousness was negatively correlated with both the mean self-risk and mean peer-risk judgments, it was essentially uncorrelated with the difference between these two variables, that is, with the optimistic bias $(r=-.07, p=.77)$.

We must be cautious about drawing conclusions from these results without further research, but one plausible interpretation of them is as follows. Perceived event seriousness is negatively related to all three kinds of risk judgments studied here: comparative judgments, self-risk judgments, and peer-risk judgments. Although perceived seriousness is partially confounded with perceived frequency-possibly because it is affected by perceived frequency - it also accounts for some variance in people's risk judgments when perceived frequency is controlled. For comparative risk judgments, this means that there is a positive relationship between perceived seriousness and the magnitude of the optimistic bias. For absolute risk judgments, there is no relationship between perceived event seriousness and the magnitude of the optimistic bias because it bears roughly the same negative relationship to both self-risk and peer-risk judgments and therefore does not affect the difference between them. By contrast, perceived event frequency is more strongly related to peer-risk judgments than to self-risk judgments, which is why there is a positive relationship between perceived event frequency and the optimistic bias. This highlights the importance of distinguishing between variables affecting people's personal risk judgments and variables affecting the optimistic bias. A given variable might have strong effects on the former but weak or null effects on the latter.

\section{GENERAL DISCUSSION}

The primary results of the current studies are quite clear. The direction of the relationship between the perceived frequency of an event and the magnitude of the optimistic bias depends on the method used to elicit people's risk judgments. When people make a single comparative risk judgment, events that are perceived as more frequent are associated with a smaller optimistic bias. When people make two absolute risk judgments-one about themselves and one about their average peer-events that are perceived as more frequent are associated with a larger optimistic bias. These results follow from the STF model of comparative risk judgments (Klar \& Giladi, 1997, 1999), the singulardistributional model of absolute risk judgments (Klar et al., 1996), and the current two-process model that integrates them.

An important implication of the current research is that many other theoretically interesting variables-in addition to perceived event frequency and seriousness-may bear different relationships to the optimistic bias as measured by comparative versus absolute risk judgments. For example, several studies have shown that the nature of the comparison other (e.g., one's average peer) affects the magnitude of the optimistic bias. It tends to be smaller when the compar- 
ison other is an individual-especially a friend or relative of the participant-and larger when the comparison other is the average member of some group (Klein \& Weinstein, 1997). Whitley and Hern (1991), for instance, found that college women judged their risk of an unwanted pregnancy to be about the same as that of their best friend, somewhat lower than that of the average college woman, and considerably lower than that of the average woman. Price (2001) has recently suggested that this might be a simple group size effect: Risk judgments increase as the size of the target group increases. The important point, however, is that according to the current analysis, this kind of effect on the optimistic bias should occur only when people make absolute risk judgments. If comparative risk judgments are not based on the specific characteristics of the comparison other, then they should not be affected by the size of that group. Consistent with this prediction, we have recently shown that the size of the optimistic bias increases as the size of the comparison group increases, but only when people make absolute risk judgments (Pentecost \& Price, 2001). The size of the comparison group has no effect on people's comparative risk judgments.

Another theoretical issue concerns the general relationship between people's beliefs about their comparative and absolute risk. Consider that Klein (1997) has shown that telling people that they are at higher or lower risk than average has a strong effect on how safe they feel. In other words, people seem to infer something about their absolute risk from information about their comparative risk. The present research, on the other hand, implies that people infer something about their comparative risk from beliefs about their absolute risk. It seems likely, therefore, that the relationship between these beliefs, and their influence on behavior, is complex. It might be, for example, that believing that they are at low absolute risk causes people to believe that they are at low comparative risk, as implied by the two-process model. Then, it might be that the belief that they are at low comparative risk causes people to feel relatively safe and to be less likely to engage in selfprotective behaviors. It might also be that this causes people to believe that they are at even lower absolute risk and so on. The dynamics of such beliefs and their effects on behavior need to be studied in more detail.

The current results also serve to remind one of the often overlooked interplay between method and theory in psychological research. It seems natural to assume that the optimistic bias in people's comparative risk judgments reflects the same underlying processes as does the optimistic bias in their absolute risk judgments. Perhaps this is why the comparative-absolute distinction has been ignored until recently. The current research, however, suggests that the judgment elicitation method actually influences the underlying psychological processes and, therefore, the nature of the optimistic bias. A similar example in the field of human judgment is research showing that probabilistic reasoning seems to improve when problems are described, and responses are made, in terms of relative frequencies rather than probabilities (Gigerenzer \& Hoffrage, 1995; Price, 1998). Likewise, survey researchers have found that the specific response scales they use can have surprisingly strong effects on how people construe and respond to survey questions (Schwarz, 1999). An important implication for research on the optimistic bias, therefore, is that new methods of eliciting people's risk judgments might produce new results. Imagine, for example, if people were simply asked about their risk of having a heart attack. Would they spontaneously compare themselves to others? Would they generate quantitative responses? These questions are definitely worth pursuing further (Klein \& Weinstein, 1997).

Finally, the relationship between method and theory is also important in considering the practical implications of the optimistic bias. Imagine, for example, a public health education campaign that seeks to provide people with more accurate information about the frequency of a particular event-to correct for a general misperception that the event is either very infrequent or very frequent. What would be the effect on the optimistic bias? The current research suggests that the effect would be either to decrease the bias or to increase it, depending on the judgment elicitation method.

\section{REFERENCES}

Alicke, M. D., Klotz, M. L., Breitenbecher, D. L., Yurak, T. J., \& Vredenburg, D. S. (1995). Personal contact, individuation, and the betterthan-average effect. Journal of Personality and Social Psychology, 68, 804-825.

Burger, J. M., \& Palmer, M. L. (1992). Changes in and generalization of unrealistic optimism following experiences with stressful events: Reactions to the 1989 California earthquake. Personality and Social Psychology Bulletin, 18, 39-43.

Cohen, J., \& Cohen, P. (1983). Applied multiple regression/correlation analysis for the behavioral sciences. Hillsdale, NJ: Erlbaum.

Gerrard, M., Gibbons, F. X., \& Bushman, B. J. (1996). Relation between perceived vulnerability to HIV and precautionary sexual behavior. Psychological Bulletin, 119, 390-409.

Gigerenzer, G., \& Hoffrage, U. (1995). How to improve Bayesian reasoning without instruction: Frequency formats. Psychological Review, 102, $684-704$.

Ginossar, Z., \& Trope, Y. (1987). Problem solving in judgment under uncertainty. Journal of Personality and Social Psychology, 52, 464 474.

Harris, P. (1996). Sufficient grounds for optimism? The relationship between perceived controllability and optimistic bias. Journal of Social and Clinical Psychology, 15, 9-52.

Jemmott, J. B., Croyle, R. T., \& Ditto, P. H. (1988). Commonsense epidemiology: Self-based judgments from laypersons and physicians. Health Psychology, 7, 55-73.

Jemmott, J. B., Ditto, P. H., \& Croyle, R. T. (1986). Judging health status: Effects of perceived prevalence and personal relevance. Journal of Personality and Social Psychology, 5, 899-905. 
Kahneman, D., \& Tversky, A. (1973). On the psychology of prediction. Psychological Review, 80, 237-251.

Klar, Y., \& Giladi, E. E. (1997). No one in my group can be below the group's average: A robust positivity bias in favor of anonymous peers. Journal of Personality and Social Psychology, 73, 885-901.

Klar Y., \& Giladi, E. E. (1999). Are most people happier than their peers, or are they just happy? Personality and Social Psychology Bulletin, 25, 585-594.

Klar, Y., Medding, A., \& Sarel, D. (1996). Nonunique invulnerability: Singular versus distributional probabilities and unrealistic optimism in comparative risk judgments. Organizational Behavior and Human Decision Processes, 67, 229-245.

Klein, W. M. (1997). Objective standards are not enough: Affective, self-evaluative, and behavioral responses to social comparative information. Journal of Personality and Social Psychology, 72, 763-774.

Klein, W. M., \& Weinstein, N. D. (1997). Social comparison and unrealistic optimism about personal risk. In B. Buunk \& F. X. Gibbons (Eds.), Health, coping, and well being: Perspectives from social comparison theory (pp. 25-61) Mahwah, NJ: Erlbaum.

Kruger, J. (1999). Lake Wobegone be gone! The "below-average effect" and the egocentric nature of comparative ability judgments. Journal of Personality and Social Psychology, 77, 221-232.

McKenna, F. P. (1993). It won't happen to me: Unrealistic optimism or illusion of control? British Journal of Psychology, 84, 39-50.

Otten, W., \& Van der Pligt, J. (1996). Context effects in the measurement of comparative optimism in probability judgments. Journal of Social and Clinical Psychology, 15, 80-101.

Pentecost, H. C., \& Price, P. C. (2001, May). A group size effect on personal risk judgment. Poster presented at the annual meeting of the Western Psychological Association, Maui, HI.

Price, P. C. (1998). Effects of a relative-frequency elicitation question on likelihood judgment accuracy: The case of external correspondence. Organizational Behavior and Human Decision Processes, 76, 277-297.

Price, P. C. (2001). A group size effect on personal risk judgments: Implications for unrealistic optimism. Memory \& Cognition, 29, 578586.

Rothman, A. J., Klein, W. M., \& Weinstein, N. D. (1996). Absolute and relative biases in estimations of personal risk. Journal of Applied Social Psychology, 26, 1213-1236.

Schwarz, N. (1999). Self-reports: How the questions shape the answers. American Psychologist, 54, 93-105.

Tversky, A., \& Kahneman, D. (1983). Extensional versus intuitive reasoning: The conjunction fallacy in probability judgment. Psychological Review, 90, 293-315.

Voth, R. D., \& Price, P. C. (1999). Reasons for unrealistic optimism in personal risk judgments. Unpublished manuscript.

Weinstein, N. D. (1980). Unrealistic optimism about future events. Journal of Personality and Social Psychology, 39, 806-820.

Weinstein, N. D. (1987). Unrealistic optimism about susceptibility to health problems: Conclusions from a community-wide sample. Journal of Behavioral Medicine, 10, 481-500.

Weinstein, N. D., \& Nicolich, M. (1993). Correct and incorrect interpretations of correlations between risk perceptions and risk behaviors. Health Psychology, 12, 235-245.

Whitley, B. E., \& Hern, A. L. (1991). Perceptions of vulnerability to pregnancy and the use of effective contraception. Personality and Social Psychology Bulletin, 17, 104-110. 